\title{
MAPS OF THE INTERVAL WITH CLOSED PERIODIC SET
}

\author{
ZBIGNIEW NITECKI ${ }^{1}$
}

\begin{abstract}
We show that for any continuous map of the interval whose periodic points form a closed set, every nonwandering point is periodic with least period a power of two.
\end{abstract}

Block showed [B1] that if the set $\operatorname{Per}(f)$ of periodic points for a continuous map of the interval $f: I \rightarrow I$ is finite and consists only of fixed points, then the nonwandering set $\Omega(f)$ equals $\operatorname{Per}(f)$. Coven and Hedlund $[\mathbf{C H}]$ extended this, obtaining the same conclusion from the weaker hypothesis that some power $g=f^{n}$ of $f$ simultaneously fixes all the periodic points. Other results related to these are established in [B2, CH, L]. In this paper, we extend the results stated above.

THEOREM. If $f: I \rightarrow I$ is continuous and $\operatorname{Per}(f)$ is a closed set, then $\Omega(f)=$ $\operatorname{Per}(f)$.

I would like to thank Ethan Coven and Louis Block for useful conversations about this problem, including a gap in my original version. I have heard recently of an independent proof of the theorem above by Jin-Cheng Xiong [X].

We note that $\operatorname{Per}(f)$ closed does not imply that the set of least periods is finite, so that Coven-Hedlund's result need not apply. To construct an example, we simply string together maps $f_{n}:\left[\frac{1}{n}, \frac{1}{n-1}\right] \rightarrow\left[\frac{1}{n}, \frac{1}{n-1}\right]$ so that $f_{n}\left(\frac{1}{k}\right)=\frac{1}{k}$ and $\operatorname{Per}\left(f_{n}\right)$ contains points of least period $2^{n}$, but none higher. We will see from the proof of our theorem that this example is in essence the only situation in which $\operatorname{Per}(f)$ is closed and $[\mathbf{C H}]$ does not apply.

Our point of departure is Block's homoclinic point theorem [B3]. Given a periodic point $p$ for $f$ and a power of $f, g=f^{n}$, define the full, left, and right unstable sets of the $g$-orbit of $p$ by

$$
\begin{aligned}
W^{u}(p, g) & =\bigcap_{\epsilon>0} \bigcup_{k>0} g^{k}(p-\epsilon, p+\epsilon), \\
W^{u}(p, g, L) & =\bigcap_{\epsilon>0} \bigcup_{k>0} g^{k}(p-\epsilon, p], \\
W^{u}(p, g, R) & =\bigcap_{\epsilon>0} \bigcup_{k>0} g^{k}[p, p+\epsilon) .
\end{aligned}
$$

It is clear that when $g(p)=p$, each unstable set is an interval containing $p$ (and perhaps nothing else). One can see that

$$
W^{u}(p, g)=W^{u}(p, g, L) \cup W^{u}(p, g, R)
$$

Received by the editors August 25, 1981.

1980 Mathematics Subject Classification. Primary 58F20, 54H20.

Key words and phrases. Maps of the interval, periodic points, nonwandering points, homoclinic points.

${ }^{1}$ Supported in part by NSF grant MCS-8102122. 
When $p \in \operatorname{Per}(f)$ has an $f$-orbit consisting of $n$ points, we number these so that $p_{1}<p_{2}<\cdots<p_{n}$. It is possible to choose sides $R_{i}$ and $L_{i}$ at each point $p_{i}$ so that

$$
\begin{aligned}
& W^{u}(p, f, R)=\bigcup_{i=1}^{n} W^{u}\left(p_{i}, f^{n}, R_{i}\right), \\
& W^{u}(p, f, L)=\bigcup_{i=1}^{n} W^{u}\left(p_{i}, f^{n}, L_{i}\right)
\end{aligned}
$$

and, whenever $p_{j}=f\left(p_{i}\right)$,

$$
\begin{aligned}
& f\left[W^{u}\left(p_{i}, f^{n}, R_{i}\right)\right]=W^{u}\left(p_{j}, f^{n}, R_{j}\right), \\
& f\left[W^{u}\left(p_{i}, f^{n}, L_{i}\right)\right]=W^{u}\left(p_{j}, f^{n}, L_{j}\right) .
\end{aligned}
$$

For further details on this, see [CN, N], noting that the unstable sets $U$ defined there are the closure of the corresponding sets $W^{u}$ defined above.

Following Block [B3], a (strong) homoclinic point for $p \in \operatorname{Per}(f)$ is a point $x \neq$ $p, x \in W^{u}(p, g)$ for which $g^{k}(x)=p$, where $g$ is a power of $f$ fixing $p$. A weak homoclinic point is defined as above, but with $g$ replaced by $f$. The following is an elaboration of Block's homoclinic point theorem [B3, Theorem A], which asserts the equivalence of the first two conditions.

PROPOSITION. The following are equivalent for $f: I \rightarrow I$ continuous.

(i) Each $p \in \operatorname{Per}(f)$ has period a power of 2 .

(ii) No $p \in \operatorname{Per}(f)$ possesses a strong homoclinic point.

(iii) No $p \in \operatorname{Per}(f)$ possesses a weak homoclinic point.

The equivalence of (i) and (ii) is Block's theorem, and (ii) $\Rightarrow$ (iii) is obvious. It is possible for a map to possess weak homoclinic points which are not themselves strong homoclinic; one example is the map described in $\S 1$ of $[\mathbf{C N}]$, where $f^{2}\left(x_{0}\right)=$ $q=f^{2}(q), p=f(q)$ and $x_{0} \in W^{u}\left(p, f^{2}, L\right) \subset W^{u}(q, f, R)$, but $x_{0} \notin W^{u}\left(q, f^{2}\right) \subset$ $[p, 1]$.

However, the existence of weak homoclinic points implies the existence of strong ones, as shown in the following proposition, from which (iii) $\Rightarrow$ (ii) and hence the proposition follows.

LEMMA 1. If the $f$-orbit of $p \in \operatorname{Per}(f)$ has a weak homoclinic point, then some point on this orbit has a strong homoclinic point.

PROOF. We first make a few observations concerning the unstable sets of periodic points with no strong homoclinic points. These arguments adapt proofs from [B2], which were formulated there with different hypotheses.

Suppose $g=f^{n}, g(p)=p$, and $p$ possesses no strong homoclinic points.

Claim 1. $W^{u}(p, g) \cap\{x \mid x>p\} \subset W^{u}(p, g, R)$.

To see this, suppose $x>p$ belongs to $W^{u}(p, g, L) \backslash W^{u}(p, g, R)$. Since $x \notin$ $W^{u}(p, g, R)$, we can find a right neighborhood of $p, V=[p, p+\epsilon]$, such that $f^{n}(v)<x$ for all $v \in V, n \geq 0$. By continuity of $f$, there exists $q<p$ such that $f(r)<p+\epsilon$ for all $r \in[q, p]$. Now, since $x \in W^{u}(p, g, L)$, there exist points $z<p$, arbitrarily near $p$, such that $f^{n}(z)=x$ for some $n=n(z)>0$. Note that for any such $z$ and $n, f^{k}(z) \notin V$ for $k=0,1, \ldots, n$. Thus, we can find $k<n$ such that $f^{k-1}(z)<p, f^{k}(z)<q$, and $f^{k+1}(z)>p+\epsilon$. Denote by 
$J$ the interval with endpoints $f^{k-1}(z)$ and $f^{k}(z)$. On one hand, by definition $p \in$ $\left(f^{k}(z), f^{k+1}(z)\right) \subset F(J)$, so some $s \in J$ satisfies $f(s)=p$. On the other hand, since $z$ can be chosen arbitrarily near $p$ from below, $q \in W^{u}(p, q, L)$. But then if $z>q, J \subset W^{u}(p, g, L) \backslash\{p\}$ and so $s$ is a strong homoclinic point for $g$.

Claim 2. If $p<q$ are fixed points of $g$ such that $q \in W^{u}(p, g, R)$ and $p \in$ $W^{u}(q, g, L)$ then $p$ and $q$ each possess a strong homoclinic point.

To see this, note that for each $\epsilon>0$ there exists $n$ such that

$$
q \in g^{n}(p, p+\epsilon), \quad p \in g^{n}(q-\epsilon, q) .
$$

But then $(q-\epsilon, q) \subset g^{n}(p, p+\epsilon)$, and $(p, p+\epsilon) \subset g^{n}(q-\epsilon, q)$ and hence

$$
q \in g^{2 n}(q-\epsilon, q), \quad p \in g^{2 n}(p, p+\epsilon) .
$$

Since $\epsilon>0$ is arbitrary, this produces strong homoclinic points.

Claim 3. Suppose $f^{n}(p)=p$ and $p \neq q=f^{k}(p)$. If the $f$-orbit of $p$ possesses no strong homoclinic orbits, then $q \notin W^{u}\left(p, f^{n}\right)$.

Again, we number the points of the $f$-orbit of $p$ so that $p_{1}<p_{2}<\cdots<$ $p_{n}$. It is clear from (3) above that if $W^{u}\left(p, f^{n}\right)$ includes $p_{i} \neq p$, then every $W^{u}\left(p_{j}, f^{n}\right)$ includes some $p_{k} \neq p_{j}$. For $j=1$, this implies (using Claim 1) that $p_{2} \in W^{u}\left(p_{1}, f^{n}, R\right)$. Then, by Claim $2, p_{1} \notin W^{u}\left(p_{2}, f^{n}\right)$. Hence, $p_{3} \in W^{u}\left(p_{2}, f^{n}, R\right)$ and inductively $p_{i+1} \in W^{u}\left(p_{i}, f^{n}, R\right)$ for $i=1, \ldots, n-1$. In particular,

$$
p_{n} \in W^{u}\left(p_{n-1}, f^{n}, R\right) \text {. }
$$

But by the left-handed analogue of Claim 1, since some $p_{i}$ to the left of $p_{n}$ must belong to $W^{u}\left(p_{n}, f^{n}\right)$, we have $p_{n-1} \in W^{u}\left(p_{n}, f^{n}, L\right)$. This together with the preceding inclusion gives a strong homoclinic point by Claim 2.

Proof of Proposition. Given Claims 1-3, the lemma is now easy to prove. Suppose $x$ is a weak homoclinic point of the $f$-orbit $\left\{p_{i} \mid i=1, \ldots, n\right\}$. Thus, for some $i, p_{i} \neq x \in W^{u}\left(p_{i}, f^{n}\right)$, and $f^{k}(x)=p_{i}$. This second condition can be replaced by $f^{k n}(x)=p_{j}$. If $i=j, x$ is a strong homoclinic point of $p_{i}$. If $i \neq j$, then by (3),

$$
p_{j} \in f^{k n} W^{u}\left(p_{i}, f^{n}\right)=W^{u}\left(p_{i}, f^{n}\right)
$$

since $f^{k n}\left(p_{i}\right)=p_{i}$. But then by Claim 3 , with $q=p_{j}, p=p_{i}$, we see that the orbit of $p_{i}$ possesses strong homoclinic points.

Recall that if (i) or (ii) fails there exists an invariant subset for $f$ with a nontrivial (one-sided) subshift of finite type as a factor [B3, BGMY, N]. Since the semiconjugacy is finite-to-one and the periodic points are dense in the phase space of the subshift but not equal to it, we have

COROLlaRY. If $f: I \rightarrow I$ is continuous with $\operatorname{Per}(f)$ closed, no $p \in \operatorname{Per}(f)$ has a homoclinic point (weak or strong).

Up to the last step, our argument for the theorem will be based on an analysis of the nonwandering set, assuming no homoclinic points. Recall the following fact:

LEMMA $2[\mathbf{C N}]$. If $f: I \rightarrow I$ is continuous and $x \in \Omega(f)$, there exist points $x_{n} \rightarrow x$ such that $f^{n}\left(x_{n}\right)=x$ for a subsequence.

A consequence is

LEMMA 3. If $f: I \rightarrow I$ is continuous and has no homoclinic points, then any $x \in \Omega(f) \backslash \operatorname{Per}(f)$ has an infinite orbit. 
Proof. If $x$ has a finite orbit then $f^{k}(x)=p \in \operatorname{Per}(f)$ for some $k>0$. Since $f^{k}\left(x_{n}\right) \rightarrow f^{k}(x)=p$ in Lemma $2, f^{n-k}\left(f^{k}\left(x_{n}\right)\right)=x$ for a subsequence implies $x \in W^{u}(p, f)$. But $x \in \operatorname{Per}(f)$ implies $x \neq p$, hence $x$ is a homoclinic point for $p$.

Maps with no homoclinic points can also be characterized by separation of orbits. We recall the formulation of this idea from [N], which summarizes versions found earlier in [B4, M, LMPY]. A set $P \subset I$ is separated to order one under $f$ if there exists a point $z$ such that for every $p \in P, f(p) \geq z$ iff $p \leq z$. For $P$ an orbit segment $\left\{x, f(x), \ldots, f^{k}(x)\right\}$, this means all even iterates (up to $k$ ) are on one side of $z$ while all odd iterates are on the other. We can always take $z$ in this definition to be fixed under $f$.

If $P$ is separated to order one under $f$, we denote the two sets into which $z$ divides $P$ by $P_{0}, P_{1}$. By induction, define $P$ to be separated to order $n$ under $f$ if $P$ is separated to order $(n-1)$ under $f$ and each half $P_{i}(i=0,1)$ is separated to order one under $f^{2}$.

We note the following without proof:

LEMMA 4. Suppose $f: I \rightarrow I$ is continuous and has no homoclinic points. Then

(i) ([LMPY], cf. [N]) if $f^{k}(y) \leq y<f(y)$ then $P=\left\{y, f(y), \ldots, f^{k-1}(y)\right\}$ is separated to order one under $f$;

(ii) ([B3], cf. [N]) in the case above, the separating point $z$ can be chosen so that $f^{2}(z)=z$ and $y \in W^{u}\left(z, f^{2}\right)$

(iii) ([M], cf. $[\mathbf{N}])$ if any set $P$ is separated to order two, then for each $i=0,1$, the convex hull of $P_{i}$ contains no fixed point of $f$.

An immediate consequence of Claim 3 in the proof of Lemma 1 is

REMARK. Suppose $p \in \operatorname{Per}(f)$ has period $n$ and no homoclinic points. If $x, y \in$ $W^{u}\left(p, f^{n}\right)$ lie on the same side of $p$, then for each $k, f^{k}(x)$ and $f^{k}(y)$ also lie on the same side of any point in the $f$-orbit of $p$.

This is because $[x, y] \subset W^{u}\left(p, f^{n}\right)$ and hence none of its images can hit a point on the $f$-orbit of $p$. Combining the Remark and Lemma 4, we prove

LEMMA 5. Fix $n>0$. If $f: I \rightarrow I$ is continuous and has no homoclinic points, and if $x \in \Omega(f) \backslash \operatorname{Per}(f)$, then

(i) the $f$-orbit of $x$ is separated to order $n$ under $f$,

(ii) $x \in \Omega\left(f^{m}\right), m=2^{n}$.

Proof. We use induction on $n$ : the inductive step consists of replacing $f$ with $f^{2}$. Thus we need only prove the case $n=1$.

Note that if a point $y$ with infinite orbit satisfies (i) of Lemma 4 for some $k \geq 2$, then by (ii) of Lemma 4 the orbit segment up to $f^{k-1}(y)$ is separated by some $z$ satisfying $f^{2}(z)=z$ and $y \in W^{u}\left(z, f^{2}\right)$. It follows that $f^{i}(y) \in W^{u}\left(z, f^{2}\right)$ for $i<k$ even and $f^{i}(y) \in W^{u}\left(f(z), f^{2}\right)$ for $i<k$ odd. Since the initial orbit segment is separated by $z$, the Remark shows that every even iterate of $y$ lies on the same side of $z$ as $y$, and every odd iterate lies on the other side of $z$. This guarantees that the whole orbit is separated by $z$.

Now fix $x \in \Omega(f) \backslash \operatorname{Per}(f)$ and pick $y$ (using Lemma 2) so near $x$ that no fixed point $z$ of $f^{2}$ lies between $f^{i}(x)$ and $f^{i}(y)$ for $i=0,1,2$, and furthermore $f^{N}(y)=$ $x$ for some $N \gg 3$. We claim some orbit segment of $y$ satisfies the hypothesis of (i) in Lemma 4. 
Let $y_{1}<y_{2}<y_{3}$ be the distinct points $y, f(y)$ and $f^{2}(y)$; the corresponding iterates of $x$ are $x_{1}<x_{2}<x_{3}$. Then $x_{1}<y_{2}<x_{3}$, and we can assume without loss of generality that $f\left(y_{2}\right)>y_{2}$. Since $x$ belongs to the orbit of $y_{2}$, so does $x_{1}$, and we have

$$
f^{M}\left(y_{2}\right)=x_{1}<y_{2}<f\left(y_{2}\right)
$$

for some $M \gg 3$. Thus by (i) of Lemma 4 and the argument above, the orbit of $y_{2}$ (including the orbit of $x$ ) is separated to order one under $f$ by a point $z$ for which $f^{2}(z)=z$ and $y_{2} \in W^{u}\left(z, f^{2}\right)$. Since $x_{2}$ and $y_{2}$ (by the choice of $y$ ) lie on one side of $z$ and both belong to $W^{u}\left(z, f^{2}\right)$, the Remark gives us that $f^{N}\left(y_{2}\right)=x_{2}$ implies $N$ even. But if $f^{N}(y)=x$, then $f^{N}\left(y_{2}\right)=x_{2}$.

Thus, we have shown that, if $x_{n} \rightarrow x$ as in Lemma 2, then every $n$ for which $f^{n}\left(x_{n}\right)=x$ is even. Thus $x \in \Omega\left(f^{2}\right)$. We have thus established the case $n=1$ of our lemma, and hence by the induction argument at the beginning, the whole result.

The final step in our argument is

LEMMA 6. If some infinite orbit is separated to all orders under $f$, then there exists a sequence of points $p_{n} \in \operatorname{Per}(f)$ converging to $q \notin \operatorname{Per}(f)$.

Proof. From the Corollary to Lemma 1, we can assume that $f$ has no homoclinic points.

Let $P$ be the infinite orbit, and $P_{0}, P_{1}$ the two halves into which it is separated by $z$ with $f(z)=z$. By (iii) of Lemma 4 , the convex hull $J_{0}$ of $P_{0}$ contains no fixed points of $f$. Since $P_{0}$ is separated to all orders under $f^{2}$, the convex hull of each of its halves contains no fixed point of $f^{2}$. We can pick $J_{1}$ to be the convex hull of the half of $P_{0}$ further from $z$, so that $\bar{J}_{1}$ contains no fixed points of $f$.

Proceeding by induction, we find a sequence of nonempty intervals $J_{i}$ with $\bar{J}_{i} \subset$ $J_{i-1}$ so that $\bar{J}_{i}$ contains no fixed points of $f^{m}, m=2^{i-1}$, but does contain fixed points for $m=2^{i}$. Thus

$$
J_{\infty}=\bigcap \bar{J}_{i}
$$

is a nonempty closed interval containing no periodic points, but at least one endpoint is a limit of periodic points $p_{n} \in \bar{J}_{n}$.

PROOF OF THEOREM. If $f: I \rightarrow I$ is continuous and $\operatorname{Per}(f)$ is closed, then by the Corollary, $f$ possesses no homoclinic points. Suppose $\Omega(f) \neq \operatorname{Per}(f)$. Pick $x \in \Omega(f) \backslash \operatorname{Per}(f)$. By Lemma 3, $x$ has an infinite orbit. Thus, by Lemma 5 (and induction on $n$ ) the $f$-orbit of $x$ is separated to all orders. But then by Lemma 6 , $\operatorname{Per}(f)$ is not closed.

\section{REFERENCES}

[B1] L. Block, Mappings of the interval with finitely many periodic points have zero entropy, Proc. Amer. Math. Soc. 67 (1977), 357-360.

[B2] _ Continuous maps of the interval with finite nonwandering set, Trans. Amer. Math. Soc. 240 (1978), 221-230.

[B3] _ Homoclinic points of mappings of the interval, Proc. Amer. Math. Soc. 72 (1978), 576-580.

[B4] - Simple periodic orbits of mappings of the interval, Trans. Amer. Math. Soc. 254 (1979), 391-398.

[BGMY] L. Block, J. Guckenheimer, M. Misiurewicz and L. S. Young, Periodic points and topological entropy of one dimensional maps, Lecture Notes in Math., vol. 819, Springer-Verlag, Berlin and New York, 1980, pp. 18-34. 
[CH] E. M. Coven and G. A. Hedlund, Continuous maps of the interval whose periodic points form a closed set, Proc. Amer. Math. Soc. 79 (1980), 127-133.

[CN] E. M. Coven and Z. Nitecki, Nonwandering sets of the powers of maps of the interval, Ergodic Theory and Dynamical Systems 1 (1981), 9-31.

[L] J. Llibre, Continuous maps of the circle with finitely many periodic points, Barcelona, 1979, preprint.

[LMPY] T. Li, M. Misiurewicz, G. Pianigiani and J. Yorke, Odd chaos (to appear).

[M] M. Misiurewicz, Invariant measures for continuous transformations of [0,1] with zero topological entropy, Lecture Notes in Math., vol. 729, Springer-Verlag, Berlin and New York, 1979, pp. 144-152.

[N] Z. Nitecki, Topological dynamics on the interval, Ergodic Thy. Dyn. Syst. II (A. Katok, ed.), Prog. Math., Birkhäuser, 1981 (to appear).

[X] J.-C. Xiong, Continuous self-maps of the closed interval whose periodic points form a closed set, Hofei, Anwhei, China, 1981, preprint.

Department of Mathematics, Tufts University, Medford, Massachusetts 02155 